\title{
Extending the EPC with Performance Measures
}

\author{
Birgit Korherr \\ Women's Postgraduate College for \\ Internet Technologies \\ Institute of Software Technology and \\ Interactive Systems \\ Vienna University of Technology \\ 1040 Wien, Austria \\ korherr@wit.tuwien.ac.at
}

\author{
Beate List \\ Women's Postgraduate College for \\ Internet Technologies \\ Institute of Software Technology and \\ Interactive Systems \\ Vienna University of Technology \\ 1040 Wien, Austria \\ list@wit.tuwien.ac.at
}

\begin{abstract}
The Event-Driven Process Chain (EPC) is designed for modelling business processes, but does not yet include any means for measuring the performance of a business process. Thus, we extend the metamodel of the EPC with performance measures to make them conceptually visible. The extensions are tested with an example business process.
\end{abstract}

\section{Keywords}

Business process modelling, performance measures, metamodel.

\section{INTRODUCTION}

The Event Driven Process Chain (EPC) is one of the most widely used Business Process Modelling Languages (BPMLs). Although the EPC offers notation elements for business process goals, it does not provide elements that make performance measures visible. The goal of this paper is to address this limitation

- by extending the EPC metamodel with performance measures and, by

- $\quad$ providing notation elements for them.

Goal-oriented business process modelling and business process performance measurement have become important topics in research and industry. Korherr et al. [2] extended the UML 2 activity diagram with business process goals and performance measures and, provide a mapping to BPEL. List et al. [3] developed a UML 2 profile for business process modelling that captures high-level characteristics, like goals and measures, customers, and deliverables.

We extend the EPC metamodel with performance measures, and provide the following contributions:

- $\quad$ Performance measures make the evaluation criteria for a business process conceptually visible and,

- $\quad$ provide a means to quantify business process goals.

Permission to make digital or hard copies of all or part of this work for personal or classroom use is granted without fee provided that copies are not made or distributed for profit or commercial advantage, and that copies bear this notice and the full citation on the first page. To copy otherwise, to republish, to post on servers or to redistribute to lists, requires prior specific permission and/or a fee.

SAC'07, March 11-15, 2007, Seoul, Korea.

Copyright 2007 ACM 1-59593-480-4 /07/0003...\$5.00.

\section{EPC AND PERFORMANCE MEASURES}

The EPC [4] has been developed within the framework of the Architecture of Integrated Information System (ARIS) and divides complex process models into separate views, in order to reduce the complexity. The views focus on functions, data, and the organisation, and can be handled independently as well as related to each other. The EPC metamodel extended with performance measures is described in Figure 1 in UML 2.0 class diagram notation. An EPC consists of functions, events, control flow connectors, logical operators, and additional process objects. A more detailed description of the EPC can be found in [1]

We extend the EPC metamodel by introducing a new view, the Measure View; shown in Figure 1 and highlighted in grey. It contains two core concepts, namely Measure and Process Goal. The process goal itself is a part of the EPC, and describes the specific intension of a business process. Each goal is quantified by at least one measure, and a goal can be refined by one or more sub goals. A measure is an abstract metaclass and is connected with one or more Measure Flow Connectors. It can be classified and implemented as Quality, Cost or Cycle Time. The quality can be expressed e.g., by a low number of complaints or a high customer satisfaction. Cost represents the expenses a business process requires for instance for its execution. Cycle time presents a time based measure and defines the processing duration of a business process instance, or part of it. It can be specialised as Working Time or Waiting Time. Working time represents the actual time a business process instance is being executed by a role. Waiting time shows the time the process instance is waiting for further processing. Quality and cost are in contrast to the cycle time often more focused on the type level of a process, as the required data is often not available on instance level.

Figure 2 illustrates the relationship between goals and measures in a so called goal measure tree. A goal can have several sub goals, and each goal has at least one measure. An example of the goal measure tree is shown in Figure 2. The main process goal is Good Process Performance. This goal has three sub-goals: Low Processing Costs, Short Process Duration, and High Customer Satisfaction. Furthermore each goal is refined by measures. The goal low processing costs is fulfilled, when the Average Processing Costs per Month are under 15 Euros. The measure cycle time is split up into two sub-cycle times and indicates that the process duration has to be less than four days. Moreover, the goal high customer satisfaction is achieved, if the Average Percentage of Complaints per Month is less than five percent. 


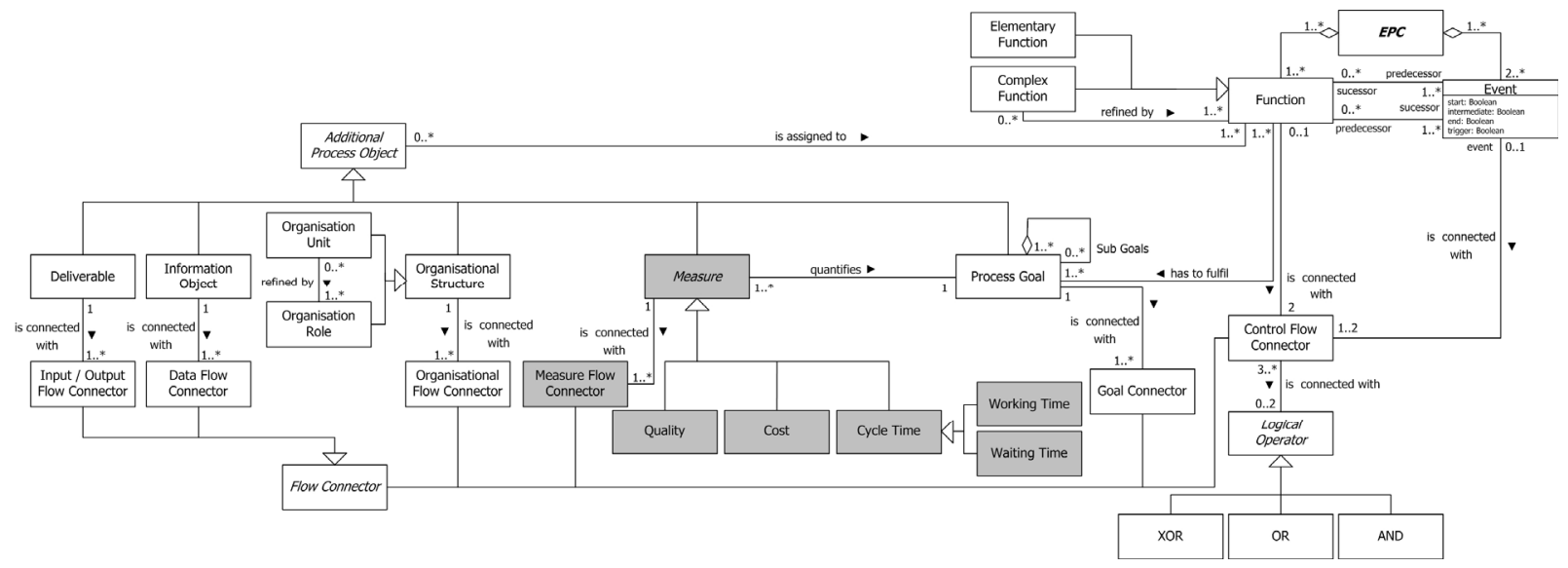

Figure 1. Extended EPC metamodel with performance measures

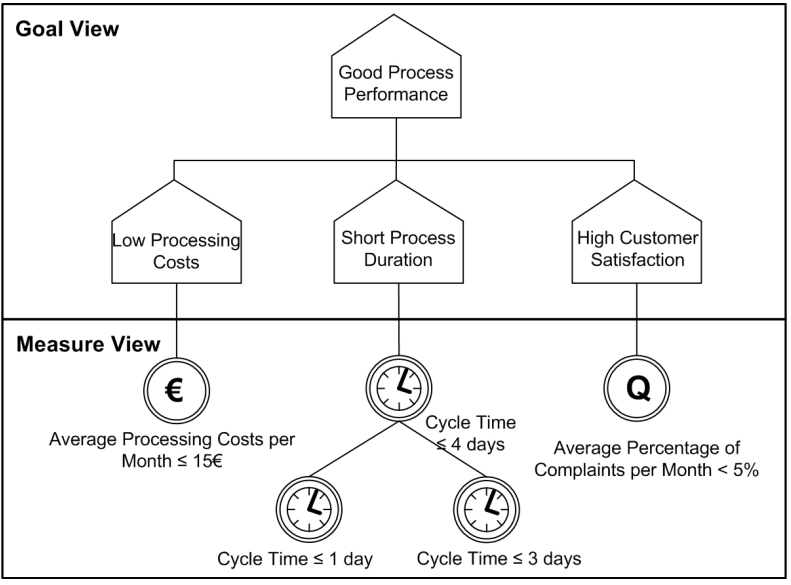

Figure 2. Goal Measure Tree

\section{EXAMPLE}

We demonstrate the practical applicability of the extension of the EPC with performance measures described in Figure 1 with a high-level business process of an insurance company, the Processing of Automobile Claims Business Process (Fig. 3). For the sake of simplicity, in Figure 3 and 4 the first and the second hierarchy levels of the business process are shown, while the finegrained levels of the business process showing the atomic activities are not described.

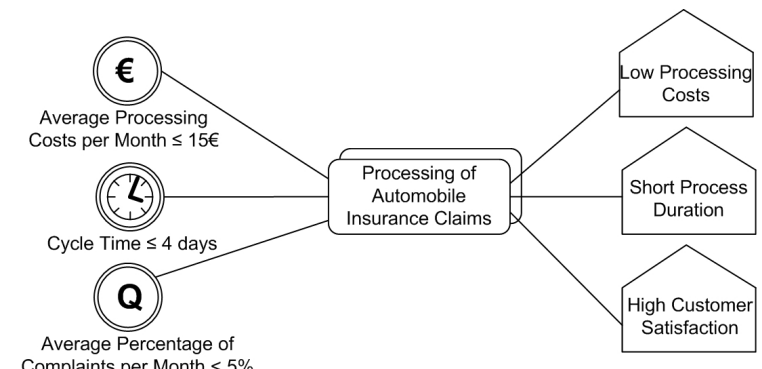
Complaints per Month $<5 \%$

Figure 3. First hierarchy level: Automobile Claims BP
The overall goal of Processing of Automobile Insurance Claims is to fulfil the process goals High Customer Satisfaction, Short Process Duration and Low Processing Costs. The process has to meet three measures, costs, cycle time and quality. The average processing costs per month have to be $15 €$ maximum, the number of complaints should not exceed five percent and, the cycle time should be not over 4 days.

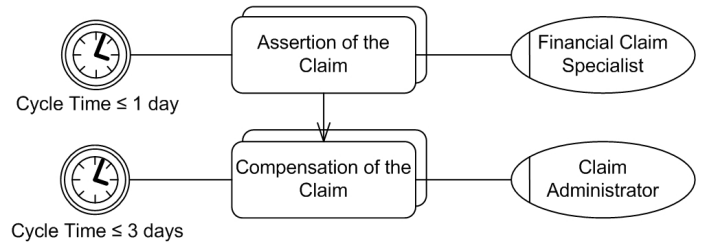

Figure 4. Second hierarchy level: Automobile Claims BP

At the second hierarchy level (Fig. 4) the organisational role Financial Claim Specialist is responsible for Assertion of the Claim. The organisational role of the Claim Administrator is responsible for the Compensation of the Claim. Furthermore assertion of the claim has to fulfil its tasks within a cycle time of one day, and compensation of the claim within three days.

\section{ACKNOWLEDGMENTS}

This research has been funded by the Austrian Federal Ministry for Education, Science, and Culture, and the European Social Fund (ESF) under grant 31.963/46-VII/9/2002.

\section{REFERENCES}

[1] Korherr, B. and List, B. A UML 2 Profile for Event Driven Process Chains, Proceedings of the 1st IFIP International Conference on Research and Practical Issues of Enterprise Information Systems, Springer Verlag, 2006.

[2] Korherr, B. and List, B. Extending the UML 2 Activity Diagram with Business Process Goals and Performance Measures and the Mapping to BPEL, Proc. of the $2^{\text {nd }}$ Int. Workshop on Best Practices of UML, Springer Verlag, 2006.

[3] List, B. and Korherr, B. A UML 2 Profile for Business Process Modelling, Proceedings of the $1^{\text {st }}$ International Workshop on Best Practices of UML, Springer Verlag, 2005.

[4] Scheer, A.-W. ARIS - Business Process Modeling. Springer Verlag, 1999 